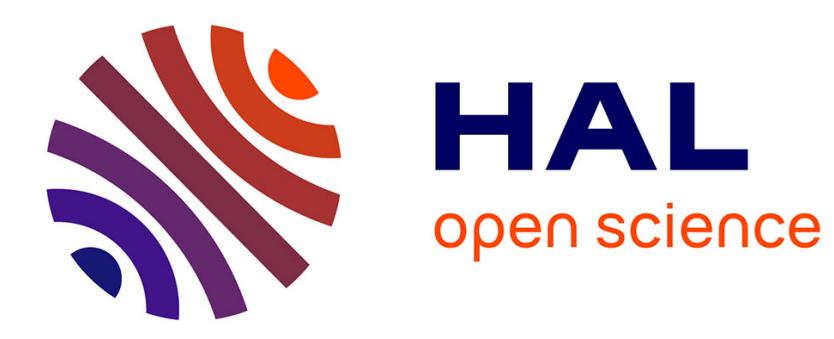

\title{
Les jardins zoologiques ou l'exotique à portée de main
}

Jean Estebanez

\section{To cite this version:}

Jean Estebanez. Les jardins zoologiques ou l'exotique à portée de main. Le Globe, 2008, 148, pp.89105. halshs-00344635

\section{HAL Id: halshs-00344635 https://shs.hal.science/halshs-00344635}

Submitted on 5 Jan 2009

HAL is a multi-disciplinary open access archive for the deposit and dissemination of scientific research documents, whether they are published or not. The documents may come from teaching and research institutions in France or abroad, or from public or private research centers.
L'archive ouverte pluridisciplinaire HAL, est destinée au dépôt et à la diffusion de documents scientifiques de niveau recherche, publiés ou non, émanant des établissements d'enseignement et de recherche français ou étrangers, des laboratoires publics ou privés. 


\section{LES JARDINS ZOOLOGIQUES OU L'EXOTIQUE A PORTEE DE MAIN}

par Jean ESTEBANEZ, Université Paris VII-Denis Diderot, département de géographie/Université de Genève, département de géographie.

Résumé : cet article montre comment l'exotique est au fondement de ce qui est présenté dans les zoos. Quelle que soit leur localisation dans le monde, le cœur de leurs collections est d'une remarquable stabilité. A travers le choix des continents représentés et le type de mise en scène des animaux, ils peuvent s'analyser comme des miniatures du monde reflétant largement le legs de la colonisation. L'exotique du zoo renvoie à un ailleurs spatial mais aussi temporel, dans un age d'or supposé, où la technologie ne s'intercalait pas entre l'homme et la nature.

Abstract : This paper shows how the exotic is a key concept to analyse zoos. Wherever their location in the world, zoos always show the same core collection. Looking at what continents and animals zoos present, we can analyse them as a form of microcosm of the world, mainly constructed through the lenses of colonisation. Zoos try to build a place that is away in space and time, at a supposed golden age when few technology was between Nature and Mankind.

Mots-clés : Exotisme; zoo; altérité ; continents; colonisation; authentique Keywords: Exotic; zoo; otherness ; continents; colonisation; authenticity

\section{Introduction}

Qui ne se souvient des lions, éléphants ou autres singes de son enfance ; ceux qu'on allait observer de temps en temps au zoo municipal ou de quelque ville lors d'un voyage familial ? De ces excursions, beaucoup plus rares sont les souvenirs de vaches, chats ou flamands roses. Les zoos ne sont ni des fermes, ni des refuges de la SPA, ni même des réserves pour la faune locale. Quelle que soit leur localisation dans le monde, le cœur de leurs collections est d'une remarquable stabilité. L'absence de félins (en particulier des lions), d'éléphants, de singes (chimpanzés, orangs-outangs, parfois gorilles) et de girafes parmi les animaux présentés par une institution remettrait en cause son statut même de zoo. De même, la présence d'animaux familiers (les chats) ou domestiques (les vaches), en dehors de la « ferme », petite partie réservée à l'intérieur du zoo, est extrêmement rare.

De la racine grecque en dehors, l'exotique renvoie à ce «qui n'est pas sur son sol naturel» (Larousse, 1982:1199), ce «qui appartient aux pays étrangers» (Larousse, 1961:848), ce «qui n'appartient pas à la civilisation de référence (celle du locuteur), et, notamment aux civilisations de l'Occident ; qui est apporté de pays lointains » (Rey, 2001:444). Pourtant, si l'exotique est directement lié à l'autre et à l'ailleurs, ce ne sont pas les objets, les lieux ou les personnes qui véhiculent intrinsèquement cette nature mais le regard qui leur est porté. Ainsi, l'exotisme est un point de vue situé et asymétrique : il se déploie hors d'Occident, en particulier à travers la colonisation, avec une prédilection pour les pays tropicaux. Par contre, la notion ne fait pas sens, par exemple pour un Mexicain qualifiant les Etats-Unis. Le regard qui exotise l'ailleurs, essentialise et simplifie l'altérité mais la juge positive, pleine de mystère et de découvertes potentielles. Le monde exotique est une sorte de cabinet de curiosité, permettant de s'échapper du quotidien à travers la contemplation d'objets, d'animaux ou de personnes ramenés d'ailleurs ou plus souvent de reproductions rappelant vaguement cet ailleurs, à travers un motif architectural, la description d'un paysage de forêt luxuriante ou un coquillage.

Le zoo est un espace de spectacle payant où le public vient découvrir une collection d'animaux essentiellement exotiques et, à l'origine, sauvages dans leur décor. Même dans les anciens pays colonisés, les zoos contemporains datent le plus souvent de la période coloniale, ou s'en inspirent largement, suivant ainsi un modèle général (il y a des éléphants et des lions au zoo d'Abidjan comme celui d'Alger, ce dernier possédant même un «village africain»). Qu'est-ce alors qu'un animal exotique ? Que penser des animaux d'ici (autochtones), le succès des mini-fermes semblant entrer en 
contradiction avec la recherche du spectacle de l'ailleurs? Par quelles autres modalités que l'origine des animaux l'exotisme se présente-t-il au zoo?

Cet article s'appuie sur trois études de cas : le zoo d'Albuquerque (Nouveau Mexique) aux EtatsUnis d'Amérique (visite le 19 mars 2008), le zoo de Bâle, en Suisse (visites le 30 novembre 2006 et le 21 février 2007) et enfin, celui de Buenos Aires, en Argentine (visite le 9 et 10 mars 2007). L'étude de terrain consiste en une ou plusieurs visites approfondies (de 4 à 6 heures) du zoo en prenant photographies et notes d'un certains nombres d'éléments prédéfinis (décors, mise en scène, conversation du public...) et de tous les éléments paraissant plus originaux. Par ailleurs, je conserve tous les prospectus et brochures distribuées par le zoo, qui donnent d'importants renseignements sur l'organisation du zoo et sur l'image que l'institution cherche à donner d'elle-même. Le zoo municipal d'Albuquerque, fondé en 1927, présente plus de 450 espèces d'animaux sur 26 hectares et a reçu environ 1'100'000 visiteurs en 2003, selon la société zoologique de Londres (2003:329), ce qui le classe comme un zoo de petite taille aux Etats-Unis. Celui de Bâle ouvre ses portes en 1874 avec 4 hectares qui sont aujourd'hui 11, compte 640 espèces et a reçu un peu mois d'un million et demi de visiteurs en 2006 (Zoological Society London, 2007:325), ce qui en fait le zoo le plus fréquenté de Suisse. Enfin le zoo de Buenos Aires, ouvert en 1875, présente 223 espèces sur 18 hectares. Ses 2,5 millions de visiteurs annuels en font le zoo le plus fréquenté d'Argentine (Zoological Society London, 2007:233). Ces trois cas, répartis dans des pays, des contextes et des statuts de villes très différents permettent d'offrir une vision relativement nuancée de la question de l'exotisme dans les zoos.

\section{Le zoo : une miniature essentiellement exotique du monde}

Les zoos d'Albuquerque, Bâle et Buenos Aires sont entourés de hauts murs ou de grilles accompagnées d'un écran de végétation. En premier lieu, ces clôtures ont une fonction sécuritaire, en rendant plus difficile la fuite des animaux, mais surtout en les protégeant des prédateurs extérieurs, que se soit des humains en quête d'un trophée ou d'une marchandise à revendre ou d'autres animaux. La deuxième fonction majeure de la clôture est de délimiter un intérieur et un extérieur, ce qui crée unité, cohérence et complétion pour le zoo. Un espace ouvert ne peut jamais réellement être parcouru et maîtrisé de manière exhaustive par un individu. Cet aspect est manifestement rassurant pour le visiteur, qui peut alors tout voir, s'il le souhaite. Cette tendance à se constituer en monde autonome n'est pas propre aux zoos mais relève de tous les parcs d'attraction. Dans les zoos, la création d'un monde en soi n'est cependant pas uniquement métaphorique, une des clés de leur organisation interne étant de plus en plus souvent un découpage par continents.

S'il est généralement admis que les pays et leurs frontières sont des constructions humaines, les continents semblent offrir un support neutre à la présentation des animaux. Or, les continents, en tant que notion, ont une histoire qui reflète les tensions et les rapports de pouvoir à l'œuvre dans le processus de connaissance et de conquête de nouveaux espaces, en particulier lors des différentes vagues de colonisation (Grataloup, 2005:235-236). Les présentations d'animaux actuelles, reflètent ainsi très souvent une vision du monde dont les origines, notamment dans la définition de l'exotique, sont à chercher dans cette période et, encore avant, dans les grandes découvertes. Le zoo d'Albuquerque utilise le découpage en continents de manière très claire, comme l'indiquent les plans distribués aux caisses et affichés tout au long du parcours. 


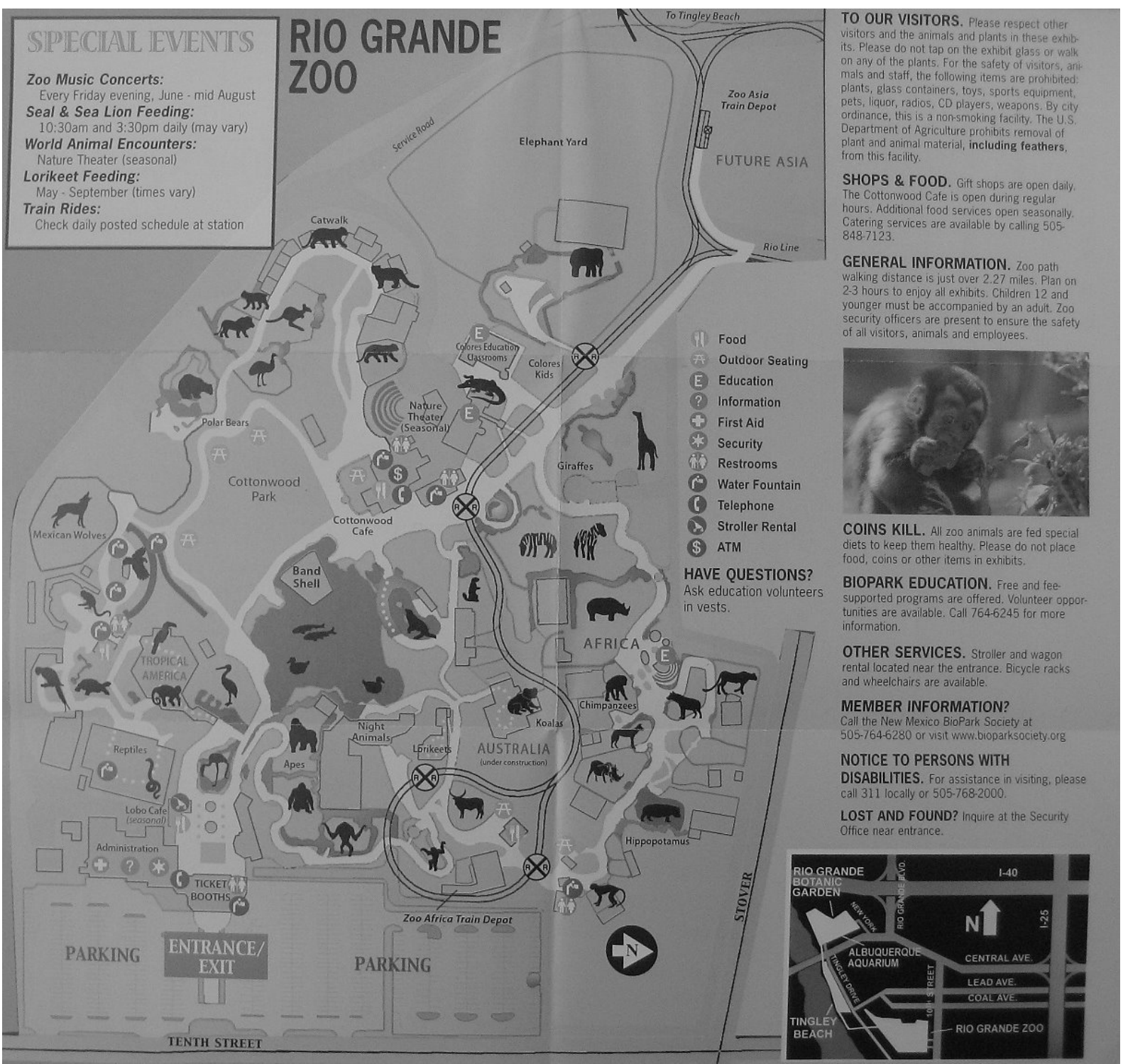

Fig 1 : Le recto de la brochure distribuée à l'entrée du zoo d'Albuquerque, photographie de l'auteur, 19 mars 2008

Les animaux sont répartis en quatre parties inégales : l'Amérique tropicale (« Tropical America ») qui couvre environ un quart de la superficie du zoo, l'Asie, en construction («Future Asia»), L'Afrique («Africa »), représentant entre le tiers et la moitié du zoo, l'Australie, en construction («Australia, under construction »), autour du koalas, l'Australie étant généralement utilisée comme le porte-étendard de l'Océanie. Enfin, environ un quart des animaux ne sont pas directement rattachés à une section, le plus souvent parce que d'anciennes logiques de classement avaient décidé de leur installation en fonction d'autres critères. La taxonomie, par exemple, peut rendre compte du regroupement de singes aux origines très variées (orangs-outangs et gorilles qui sont tous les deux à proximité immédiate de l'entrée); les économies d'échelles peuvent elles rendre compte du regroupement des espèces aquatiques, disperser de petits aquariums dans tout le parc étant très coûteux. C'est donc une miniature du monde animal qui est présentée au zoo avec toute une série de biais très notables. En premier lieu, si on suit le découpage généralement admis par continents, point d'Europe ici. L'Amérique du Nord est également exclue même s'il y a bien quelques canards sur le lac de Cottonwood Park. Ceux-ci ne sont cependant nullement contraints de rester au zoo par un système de cages ou de découpe des jointures. Attirés par la nourriture, ils ont à peu près le même statut d'animal mi-sauvage, mi-domestique que leurs congénères des jardins et parcs urbains et ne font ainsi pas réellement partie de la collection du zoo. Deuxième point important, par la surface qu'elle occupe 
dans le zoo, l'Afrique est largement surreprésentée. Cette tendance se vérifie dans la plupart des zoos, même en Afrique, sans doute parce que le continent est communément pensé comme le parangon de l'exotisme (et du sauvage). En ce sens, l'analyse du zoo nous en dit plus sur la façon de percevoir le monde des responsables de sa construction (depuis les architectes, jusqu'au jardinier, chargé de la modification du paysage) que sur les animaux qui sont présentés.

Ce regard est clairement orienté depuis un centre qui s'affiche peu en représentation (Europe puis Amérique du Nord), parce qu'il est essentiellement le même, le quotidien pour les spectateurs. Il porte sur l'altérité du monde, appréhendée principalement à travers les filtres de l'exotique. Quand le terme « exotic » apparaît en anglais, au $\mathrm{XVI}^{\mathrm{e}}$ siècle, il désigne ce qui est en-dehors (Knellwolf, 2002:10), s'appuyant dès lors sur une vision dichotomique du monde. Ce n'est que dans un second temps, à partir de la seconde vague d'exploration du XVII ${ }^{\mathrm{e}}$ et $\mathrm{XVIII}^{\mathrm{e}}$ siècle (Knellwolf, 2002:10), qu'il commence à renvoyer à l'attraction sensuelle du lointain, largement lié à ses paysages et son climat tropical, rendant simplement plus précise la définition de ce qui est en dehors. L'Afrique, l'Amérique tropicale, une partie de l'Asie et l'Océanie, au moment de sa création, deviennent ainsi des espaces exotiques.

L'entrée nord de la partie dite africaine du zoo d'Albuquerque (entre l'enclos des girafes et des zèbres) est assez caractéristique à cet égard. Des troncs d'arbres d'environ quatre mètres de haut, liés ensembles et scellés à une base en béton, forment une palissade qui masque la suite du parcours. A gauche, trois panneaux indiquent où trouver les éléphants, les girafes et les rhinocéros blancs. A droite, l'indication «Africa » avec la carte de l'Afrique, un tonneau et une sorte de totem en métal portant à la base et sur le premier cylindre des serpents, un crocodile ; un lézard sur le second et un homme avec une lance, sur le troisième. Le premier effet de la palissade est de susciter la curiosité, le désir de se lancer dans une sorte d'exploration en miniature. Le deuxième effet est de réifier l'unité de l'ensemble dit africain en lui attribuant des limites précises qui sont rappelés par la carte de l'Afrique. Cette carte, qui est plutôt une image, n'a aucune fonction didactique. Simple forme jaune, elle tient du pictogramme et sert manifestement, tout comme le totem, à dire aux visiteurs : "attention, nous entrons dans un autre espace. Nous changeons d'ambiance ». L'Afrique est un monde particulier, dans lequel on ne pénètre que par une porte, la palissade tranchant nettement entre ce qui est en dedans et ce qui est en dehors.

L'opposition entre exotique et non exotique peut aussi se décliner par la combinaison cœur civilisé/périphérie primitive dont Markula (1997:210) emprunte les concepts de base à Wallerstein. Le cœur est défini et composé par les nations majeures, c'est-à-dire essentiellement l'ensemble qui s'auto définit comme l'Europe et l'Amérique du Nord, dans le processus de constitution du capitalisme industriel. La périphérie est alors constituée par les espaces dont la productivité est appropriée par le cœur ou tout du moins, qui en sont dépendants. Les pays et les continents sont ainsi classés sur une échelle du progrès, ce que reflète le vocabulaire pays développé, pays sous-développé, pays en voie de développement. L'exotique, par la façon même dont il est structuré comme un regard sur l'altérité, ne peut être qu'un regard du cœur porté sur la périphérie. L'Europe ou l'Amérique du Nord peuvent être sauvages (dans leur périphéries internes respectives) mais pas exotiques. La superposition entre exotisme et sauvagerie ne fonctionne donc que de manière partielle, les deux termes ne connotant pas tout à fait le même type d'altérité.

Qu'en est-il alors dans les zoos où la division en continents n'est pas directement utilisée sur les plans pour classifier les animaux ? Tout d'abord, la surreprésentation de l'exotique y est tout aussi manifeste et par ailleurs il est souvent assez aisé de retracer mentalement les limites de certains d'entre eux, à commencer par l'Afrique. B. Debarbieux (1995:105) a bien montré comment certains lieux ont une fonction de structuration symbolique du territoire par un effet de synecdoque (la partie -ici le lieupour le tout, c'est-à-dire le territoire). Il me semble qu'on peut appliquer un raisonnement parallèle pour un certain nombre d'espèces dont les représentants fonctionnent comme de véritables « animauxgénériques ». Le lion, la girafe, le panda, le lama (etc.) ont une fonction typologique dans l'économie générale du zoo, en indiquant clairement à chacun où il se trouve dans la mappemonde imaginaire qui s'y déploie. Il existe ainsi un lien, établi et stabilisé sur la longue durée entre un certain nombre d'animaux et des zones de la Terre. On retrouve déjà des traces de ce lien sur les mappemondes richement illustrées de la Renaissance (Jacob, 1992:215) et il est toujours évident aujourd'hui dans les livres pour enfants. 


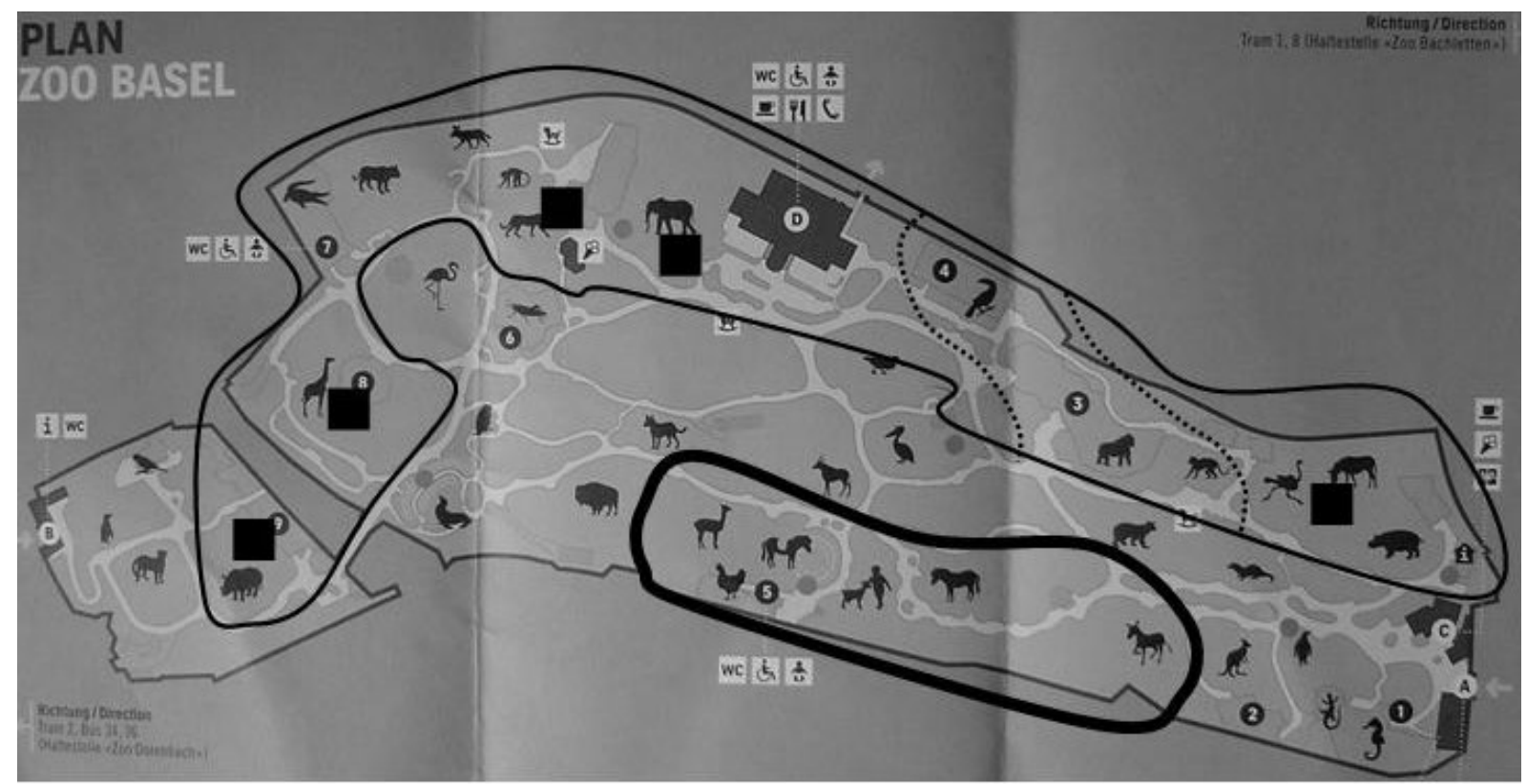

- Des animaux " africains » ? L'exotisme et la sauvagerie mis en valeur

Sous le signe de la domestication : des animaux d' «Europe »?

Les " animaux-génériques »

de 1 ' « Afrique "

Fig. 2 : Brochure retouchée présentant la carte du zoo de Bâle, photographie et schéma de l'auteur, mars 2008

La carte qui est distribuée à l'entrée du zoo de Bâle ne présente que quelques unes des 608 espèces présentes au zoo et ce sont bien celles qui font office d'icônes pour le zoo en tant que références les plus efficaces à l'ailleurs. J'ai indique par des carrés noirs, les "animaux-génériques » de l'Afrique qui sont, dans le sens des aiguilles d'une montre, les rhinocéros, girafes, lions, éléphants, zèbres, autruches et hippopotames. On peut noter qu'il existe d'autres « animaux génériques », comme le kangourou, pour l'Australie, mais ils sont en général isolés. Au contraire, dans le cas des animaux représentant l'Afrique, la série se déploie quasiment sur tout le parc. Si on y ajoute tous les animaux moins connus dont l'origine est également en africaine, on se rend compte qu'il y a une véritable continuité dans la répartition de ces animaux au zoo de Bâle. C'est cette Afrique imaginaire que j'ai délimitée par le trait fin sur la figure 2. Elle représente un peu moins de la moitié de la superficie du zoo, ce qui est d'autant plus remarquable qu'aucun autre ensemble d'importance n'apparaît en dehors d'un bloc que j'ai délimité par un trait épais et qui couvre environ un sixième de la surface totale. Tous les animaux que j'ai regroupés dans l'ensemble «Europe» sont en effet bien d'origine européenne mais il faut surtout noter qu'ils sont désignés comme «domestiques» par les panneaux qui accompagnent chaque enclos. Cette zone est la seule où on note une présence humaine à travers la figure du jeune enfant. La chèvre, animal domestique, entre quasiment dans le cercle des familiers du fait de la caresse qui lui est prodigué. Les fauves ou les éléphants renvoient les visiteurs aux images du safari quand les chèvres naines renvoient à l'image assez idéalisée et apaisante pour un citadin de la ferme. Sans que cela apparaisse de manière directe la dichotomie entre exotique/non-exotique recoupe ici partiellement l'opposition entre domestique et sauvage qui renvoient, d'un côté, à l'Europe, de l'autre à l'Afrique. Les mises en scène des zoos contribuent ainsi souvent à durcir et naturaliser des oppositions qui sont déjà admises. 


\section{Qu'est-ce qu'un animal exotique?}

Les animaux sont exotiques en tant que tels, si on entend par exotique, une forme d'altérité qui les rend curieux. Les animaux peuvent être étranges du fait d'une taille (éléphant, girafe, boas), d'un poids (éléphant, orques), d'une forme (ornithorynque), d'une couleur (certains perroquets) ou d'une dangerosité supposée (félins), jugée extraordinaire c'est-à-dire littéralement, hors du quotidien. En ce sens, on comprend que le zoo ne présente pas d'animaux familiers, qui sont précisément les animaux de la vie de tous les jours. Comment penser alors l'importance croissante des animaux d'ici (de la région ou du pays où se situe le zoo) et des animaux domestiques (chèvres ou moutons) dans les zoos? Il existe désormais de manière quasiment systématique une mini-ferme dans les zoos, où on n'hésite pas à construire un décor supposé recréer l'ambiance d'une véritable ferme avec de petits cabanons en bois reproduisant les boxes des animaux et des mangeoires. Dans un contexte général d'urbanisation et de tertiarisation des économies, les animaux, y compris domestiques, s'éloignent du quotidien de la plupart des habitants des pays riches. L'ailleurs peut finalement commencer à assez peu de distance de chez soi, dès qu'on sort de l'urbain. Les animaux autochtones ou domestiques sont de plus en plus curieux pour le public général et peuvent, sans doute pour cette raison, attirer tout autant qu'une girafe ou un lion, à partir du moment où il est possible de les toucher, ce qui n'est pas possible avec les animaux sauvages, alors même qu'il y a une forte demande de contact avec les animaux de la part du public. Même si l'attitude des animaux du zoo peut sembler artificielle à certains visiteurs, qui les pensent conditionnés par leur captivité, ils restent capables de décisions, de pensée et de mouvement autonome. En ce sens, le public reconnaît qu'ils appartiennent au même monde qu'eux, contrairement aux objets ou aux rochers, dont l'altérité est tellement grande qu'elle n'en est plus pertinente pour servir de borne à sa propre identité. Bien sûr, les animaux qui possèdent des bras, plutôt que des ailes, des poils plutôt que écailles attirent plus encore, comme le souligne l'anthropomorphisme avec lequel sont désignés les mammifères et en particulier les singes. Pourtant, le monde intérieur des animaux et la communication directe avec eux semble inaccessible, d'où la joie intense qui est manifestée quand quelqu'un est fixé du regard par un gorille ou suscité une réaction de sa part, en établissant ainsi de manière fugitive un contact. " Mais que peuvent-ils bien penser ? », ne peut-on s'empêcher de se demander. Le monde des humains et le monde des animaux semble ainsi à la fois très proche physiquement (avec quasiment une mise en contact au zoo) et physiologiquement (corps fonctionnant sur un même modèle pour les mammifères) et très loin symboliquement, créant l'altérité qu'ils portent de manière intrinsèque.

En plus de cet exotisme, au sens de curiosité, un grand nombre d'animaux du zoo sont exotiques parce qu'ils viennent d'ailleurs que l'Occident. En quoi consiste par exemple l'exotisme des éléphants du zoo de Buenos Aires? 


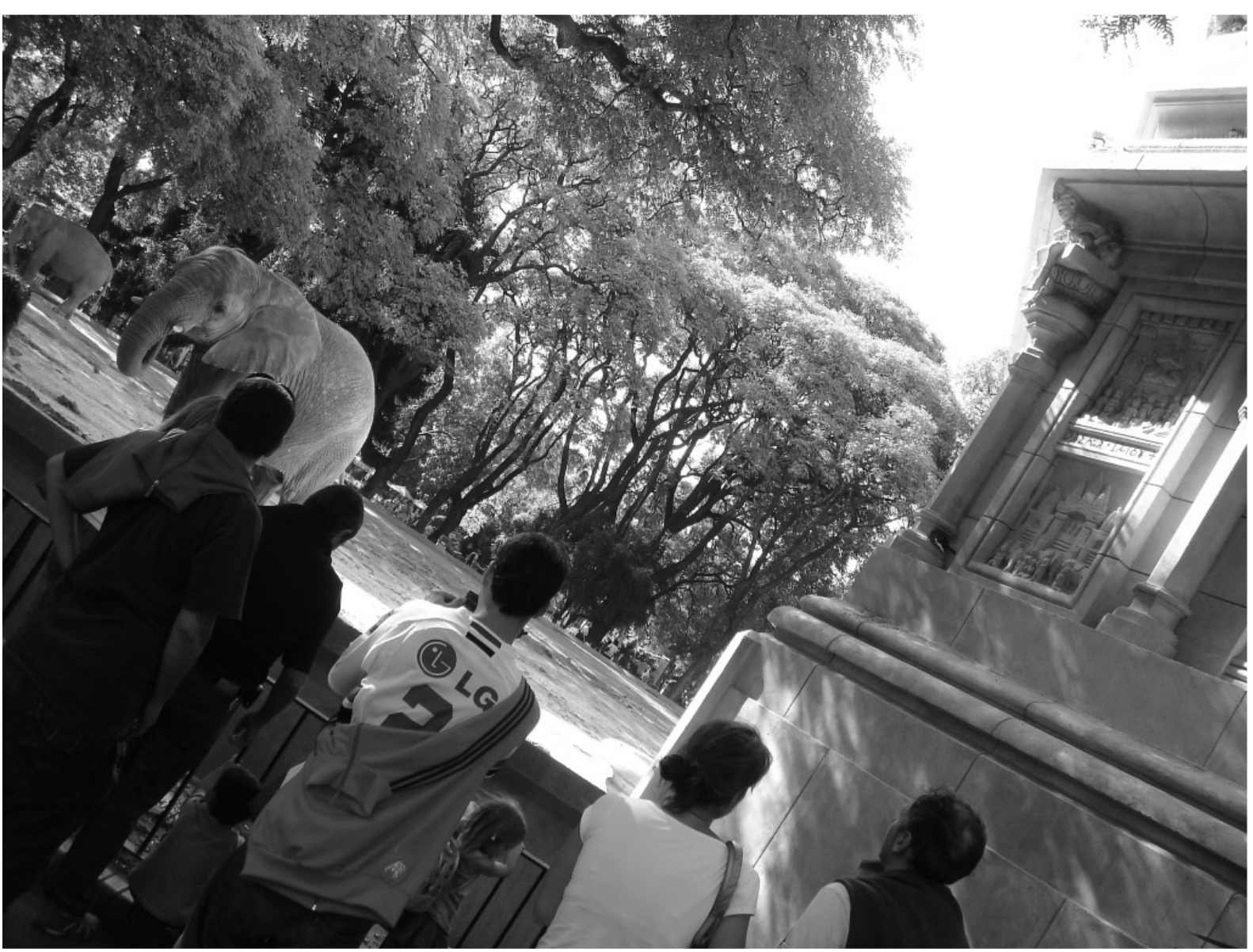

Fig 3 : Quelle est l'origine des éléphants du zoo de Buenos Aires ?, photographie de l'auteur, 10 mars 2007

Un public assez nombreux se presse autour de leur enclos. De vaste taille, il est complètement minéral. C'est un plateau de béton, bordé par une fosse de sécurité, sur lequel a été tassée une couche de terre et qui ne porte aucune plantation. Les éléphants sont donc constamment sous le regard du public, le bâtiment d'hivernage n'étant ouvert que la nuit, dans un espace neutre et à faible distance. En accord avec le discours officiel du zoo sur son rôle éducatif, l'enclos peut alors être conçu comme une page d'encyclopédie vivante, aucun élément de décor ne venant interférer avec l'observation des éléphants. Il reste alors à supposer que le public est capable d'apprendre quelque chose du simple fait d'être proche d'un éléphant. Si on entend par apprentissage l'observation d'un animal vivant, de ses mouvements, de sa taille, de sa morphologie ou encore la mise en adéquation entre le nom «éléphant » et le gros animal gris qui est dans l'enclos (ce que la plupart des parents essayent de faire comprendre par répétition à leurs jeunes enfants), la fonction éducative du zoo est avérée. Par contre, si l'on suit les objectifs même du zoo à propos de la classification des animaux, de leur répartition et biologie, l'échec est patent, quasiment personne ne prenant le temps de lire les panneaux.

Bien qu'il n'y ait aucun décor végétal ou minéral, le bâtiment d'hivernage sert en quelque sorte d'arrière-plan culturel aux animaux et renforce leur exotisme à travers une série d'éléments génériques qui connotent l'Asie en Occident. Le bâtiment évoque un temple hindou à l'aide d'une sculpture et de bas-reliefs où apparaissent des coupoles, une foule en prière, portant le turban, devant un dieu assis en position du yoga. L'objectif est de créer une atmosphère, pas de produire une reconstitution historique: les signes sculptés entre les deux bas-reliefs n'appartiennent à aucune langue et ne produisent d'autre message que celui de l'altérité. Par ailleurs, les éléphants sont eux clairement africains à en suivre la fameuse règle des grandes ou des petites oreilles... Ce mélange ne semble poser aucun problème à la direction du zoo, ni au public, qui ne s'aperçoit même pas de la contradiction interne que porte l'enclos, à en croire les quelques discussions que j'ai pu relever. Cela indique bien une connaissance faible d'éléments de zoologie de base et un désintérêt pour ces 
questions, de la part du public, mais surtout de la direction du zoo, en opposition totale avec son positionnement officiel. Dans un musée, une erreur aussi évidente (par exemple l'attribution d'un tableau de Picasso à Cézanne) ne manquerait pas d'attirer les remarques d'une partie des visiteurs et aurait de toute façon peu de chance de se produire, les conservateurs des grandes institutions étant rigoureux avec leurs attributions. C'est bien que la question des connaissances n'est vraiment pas ce qui compte au zoo.

L'éléphant, et plus généralement les animaux du zoo, sont des éléments plastiques dont le sens est largement déterminé par le paysage et l'ensemble des artefacts qui les entourent. Les singes de la Ménagerie du Jardin des Plantes à Paris paraissent moins exotiques que ceux du « JungleWorld» du zoo du Bronx parce qu'ils ne sont pas présentés dans un décor aussi élaboré. Au zoo du Bronx, le visiteur est plongé dans une ambiance de forêt tropicale qui contribue autant sinon plus que les animaux eux-mêmes à l'exotisme de la scène. A la Ménagerie, les cages d'assez petite dimension sont entièrement fermées et uniquement composées de béton et de métal. L'objectif à la toute fin du XVIII et au début du XIX ${ }^{\mathrm{e}}$ siècle était de pouvoir observer d'assez près un spécimen d'une espèce sans devoir se rendre sur son lieu de vie, avec tous les périls que cela comprenait. A cette fin, une cage sans décor et aisée à nettoyer semblait alors le compromis idéal. Cette absence de mise en scène devient alors un signe même de la fonction du singe comme objet de science.

Dans l'enclos des éléphants de Buenos Aires, l'atmosphère exotique de la scène vient certes pour part des animaux eux-mêmes, en tant que représentants de l'Afrique, mais elle est principalement créée par la juxtaposition de références incompatibles, qui détruisent toute idée de localisation réelle. L'exotique renvoie à l'imaginaire c'est-à-dire à un ailleurs qui n'existe que par la littérature, les peintures, les films ou les décors d'un zoo. Si tout le monde semble satisfait des incohérences factuelles qui truffent les enclos en terme de localisation ou d'adéquation entre les animaux, les plantes et les décors, c'est précisément parce que ce processus de décontextualisation (isoler un élément d'un ensemble)/recontextualisation (le réintroduire dans un nouvel ensemble) est un des moteurs principaux de l'exotisme (Masson, 1998:3). Se télescopent ainsi des images comme celle du safari ou du mystère de la jungle et des civilisations perdues (avec le modèle d'Angkor Vat), dont rendent notamment compte les panneaux en bas relief, où une cérémonie religieuse et des signes sensés figurer une écriture ont étés tracés. Si ces mises en scènes sont avant tout celles de l'imaginaire, elles n'en revendiquent pas moins une forme d'authenticité.

\section{L'exotique au zoo, entre authenticité et nostalgie d'un age d'or rêvé}




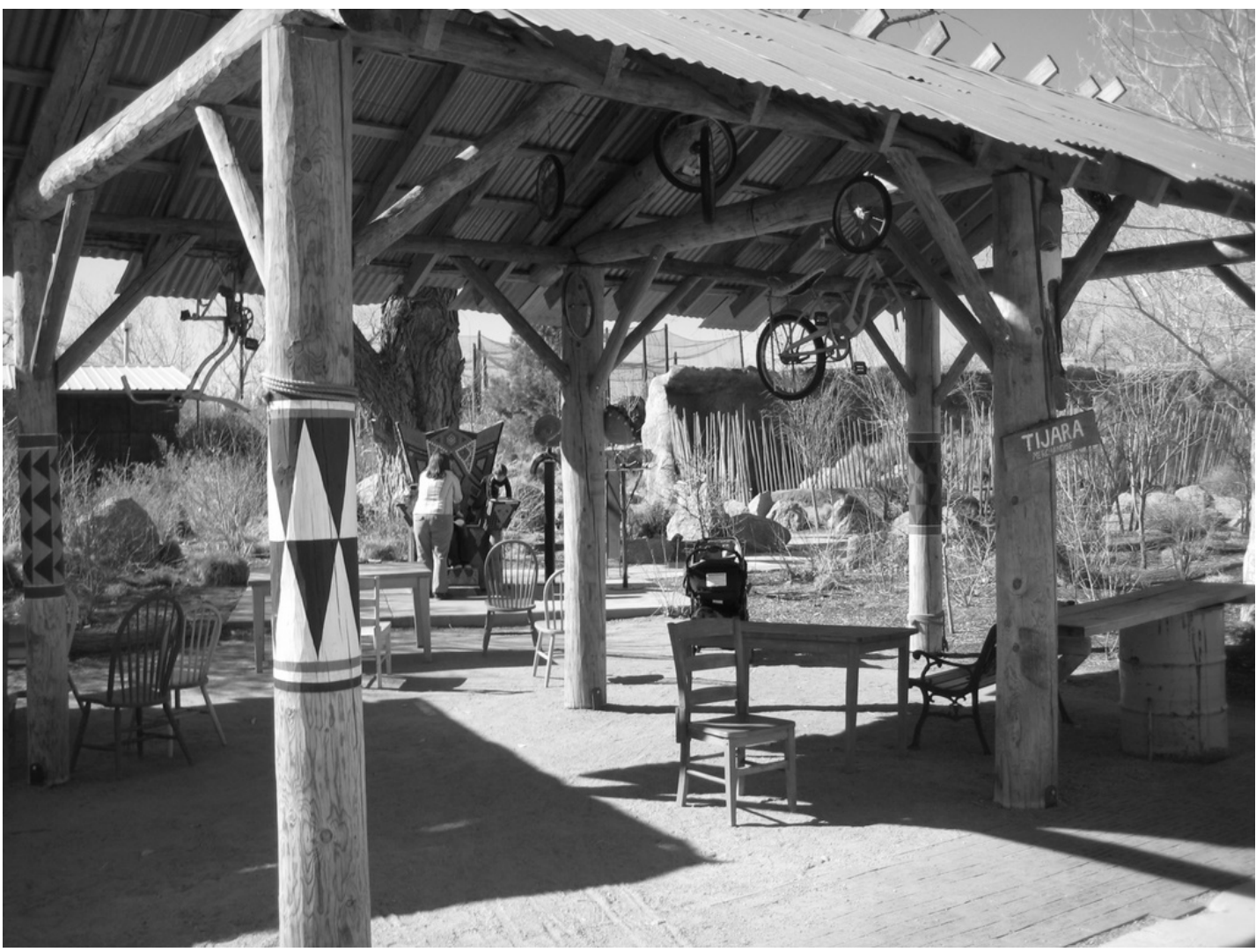

Fig 4 : L'entrée sud de la partie africaine du zoo d'Albuquerque, photographie de l'auteur, 19 mars 2008

Cette photographie présente le détail d'un groupe de constructions qui se situent à l'entrée sud de la partie africaine du zoo d'Albuquerque, ouverte au début de l'année 2008. Après voir passé la ligne du chemin de fer miniature qui traverse le parc (voir figure 1), les visiteurs arrivent sur cette sorte de place où se trouvent, à gauche, des toilettes et à droite, la reconstitution d'une boutique, où un ensemble de chaises et de tables invitent à prendre quelque repos à l'ombre, tout en contemplant le paysage. A gauche de cette boutique, un autre bâtiment de même structure, entre l'échoppe et le musée en plein air, présente des instruments de musique, dont le nom est mentionné par une étiquette. A l'arrière-plan, émergent les grillages qui forment le sommet de la cage des chimpanzés et des palissades qui délimitent l'enclos des phacochères. La boutique mesure environ 15 par 3 mètres et n'est composée que d'un toit de tôle fixé sur une armature en bois reposant sur un sol de planches et de terre battue. Les fûts qui portent le toit sont ornés de motifs géométriques et on peut observer, à droite, un comptoir constitué d'une planche reposant sur un baril de pétrole. Du toit, pendent des vélos incomplets et des pièces détachées passablement inutilisables, comme une roue sans pneu. Un panneau précise la fonction du lieu pour le public: marchandises («merchandise» soit la traduction de «Tijara »). Derrière la boutique, un trône couvert de mosaïques de couleurs, ordonnées géométriquement, est constamment utilisé comme décor par des parents qui y voient l'endroit idéal pour photographier leur progéniture. Que penser de cette mise en scène ?

Cette place fonctionne comme une sorte d'antichambre à l'Afrique en créant une atmosphère d'authenticité tribale par tout un ensemble de détails. Comme le rappelle Markula (1997:216), reprenant Eco, l'authenticité n'a pas grand-chose à voire avec des faits historiques vérifiés mais à une apparence. Si la mise en scène semble authentique, elle est authentique. L'authentique correspond ainsi beaucoup plus fortement à des bâtiments, des produits, des attitudes conformes aux stéréotypes pré-existants chez les spectateurs (Andreassen, 2003:29) qu'à une réalité avérée. L'Afrique que construit cette place est pauvre, proche de la nature, du fait de l'absence presque totale de médiation technologique et de l'omniprésence des animaux tout autour des lieux, mystérieuse dans son art qu'on suppose liés à une forme d'animisme, traditionnelle dans son système d'organisation politique (le trône). 
S'il existe sans doute des lieux qui peuvent avoir des traits communs avec cette place, ils ne représentent qu'un petit échantillon de la variété de lieux d'habitations possibles en "Afrique». Cependant, à en juger par l'absolue similarité des mises en scène qui se retrouvent tout au long du parcours africain, cette place ne cherche à reproduire aucun espace en particulier mais bien plutôt à matérialiser l'essence d'une prétendue Afrique éternelle.

L'exotisme naît, là encore, de la destruction de toute référence à une localisation réelle mais aussi de son ancrage dans un âge d'or imaginaire. L'exotique renvoie par là doublement à l'ailleurs, à travers un lieu mais aussi une époque imaginaire. Présenter un décor de ville avec de hauts buildings, des routes asphaltées ou un supermarché, en ne se conformant pas aux stéréotypes, semblerait tout simplement inauthentique (Conklin, 1997:726). Si l'Afrique, l'Amérique tropicale et une partie de l'Asie ou de l'Océanie sont des espaces exotiques, les villes modernes ne le sont pas. S'il n'y a pas d'humains dans les décors, ils n'en représentent pas moins des espaces du quotidien, censés correspondre à l'activité de ses habitants. Généralement, les activités humaines et leurs constructions sont jugées peu compatibles avec la mise en scène de la "nature », sauf s'il s'agit de civilisations anciennes, de préférences disparues, ou de populations vivant de manière «traditionnelle » et « authentique », c'est-à-dire dans une forme de passé. De fait, l'absence de représentation de l'Europe ou de l'Amérique du Nord au zoo d'Albuquerque ou sa présentation sous les formes du domestique au zoo de Bâle, est le signe d'une profonde inégalité dans les points de vues, ce qui est d'autant plus frappant que la Suisse ou les Etats-Unis n'ont jamais eu d'empire colonial en tant que tel. Ainsi, le cœur du monde est pensé comme l'espace de la modernité, qui ne peut donc pas être exotique, quand la périphérie ne peut pas être moderne, puisqu'elle est exotique.

Andreassen, R. (2003), «The 'exotic' as mass entertainment: Denmark 1878-1909», Race \& Class, vol. 45 , n. 2, pp. 21-38.

Conklin, B. A. (1997), «Body paint, feathers, and vcrs [= Video cassette recorder]: aesthetics and authenticity in Amazonian activism », American Ethnologist, vol. 24, n. 4, pp. 711-737.

Debarbieux, B. (1995), «Le lieu, le territoire, et trois figures de rhétorique », L'Espace géographique, t. 2, pp. $97-112$.

Grataloup, C. (2005), «L'identité de la carte », Communications, t. 77, pp. 235-251.

Jacob C. (1992), L'empire des cartes. Approche théorique de la cartographie à travers l'histoire, Albin Michel, Paris.

Knellwolf, C. (2002), «The Exotic Frontier of the Imperial Imagination », Eighteenth-Century Life, vol. 26, n. 3, pp. 10-30.

Larousse (1961), Grand Larousse encyclopédique en 10 volumes, Larousse, Paris.

Larousse (1982), Grand dictionnaire universel du XIXe siècle, Slatkine, Genève et Paris.

Markula, P. (1997), "As a Tourist in Tahiti : An Analysis of Personal Experience », Journal of Contemporary Ethnography, vol. 26, pp. 202-224.

Masson, P. (1998), Infelicities. Representations of the Exotic, The John Hopkins University Press, Baltimore and London.

Rey, A. (2001) dir., Le grand Robert de la langue française, Le Robert, Paris.

Zoological Society of London (2003), International Zoo Yearbook, Blackwell Publishing, Oxford.

Zoological Society of London (2007), International Zoo Yearbook, Blackwell Publishing, Oxford. 